Frederic C. Blow $\cdot$ John E. Zeber · John F. McCarthy · Marcia Valenstein • Leah Gillon · C. Raymond Bingham

\title{
Ethnicity and diagnostic patterns in veterans with psychoses
}

Accepted: 26 April 2004

Abstract Background Differential diagnosis of schizophrenia and bipolar disorder is a challenging but important task. These conditions often exhibit overlapping clinical symptomatology, but have different prognoses and pharmacological management strategies. Factors other than clinical presentation may influence diagnosis. Past studies suggest that ethnicity is one such factor, with variations observed in diagnostic rates of serious mental illness (SMI). With increasing attention paid to provider cultural competency, we investigate current diagnostic practices within a veteran population. Method Controlling for patient need characteristics and illness severity, we examine whether ethnic differences in diagnosis continue to exist. If so, race may adversely enter the evaluation process. A national database of all SMI veterans explores the relationship between ethnicity and diagnosis. The role of symptomatology is also examined. Given minimal variation in veteran socioeconomic status, the Department of Veterans Affairs (VA) provides a natural setting to address this confounding factor. The 1999 National Psychosis Registry provides a sample of 134,523 veterans diagnosed with schizophrenia,

F. C. Blow, PhD · J. F. McCarthy, PhD · M. Valenstein, MD, MS •

C. R. Bingham, $\mathrm{PhD}$

Dept. of Psychiatry

University of Michigan

Ann Arbor, Michigan

J. E. Zeber, MHA

Dept. of Health Management and Policy

University of Michigan School of Public Health

Ann Arbor, Michigan

John E. Zeber, MHA (区) • F. C. Blow, PhD · J. F. McCarthy, PhD •

M. Valenstein, MD, MS · L. Gillon, MSW

Health Services Research \& Development (HSR\&D)

Serious Mental Illness Treatment, Evaluation, and Research Center (SMITREC)

Dept. of Veterans Affairs Medical Center

P. O. Box 130170

Ann Arbor, MI 48113-0170, USA

Tel.: + 1-734/769-7100, ext 6260

Fax: + 1-734/761-2617

E-Mail: john.zeber@va.med.gov schizoaffective disorder, or bipolar disorder. Multinomial logistic regression yielded odds ratios (OR) for being diagnosed with schizophrenia versus bipolar disorder; the schizoaffective versus bipolar risk was likewise assessed, exploring theoretical aspects of a psychosis-affective 'continuum'. Results Small effects were observed for being male, single or rural resident. However, the demographic characteristic most strongly associated with a schizophrenia diagnosis was race. The OR for African Americans was 4.05, and 3.15 for Hispanics. Similar though less dramatic results were revealed for schizoaffective disorder. Conclusions This study confirms continued ethnic disparities in diagnostic patterns, and highlights the importance of recognizing ethnic differences in symptom presentation while emphasizing greater cultural competency.

Key words ethnicity - disparities - schizophrenia clinical diagnosis

\section{Introduction}

Differential diagnosis of schizophrenia and bipolar disorder is an important but difficult diagnostic task. Cross-sectionally, these two serious mental illnesses (SMI) can have similar clinical presentations; shared symptomatology includes hallucinations, delusional beliefs, disorganized speech, and affective instability. However, unlike bipolar disorder, a diagnosis of schizophrenia requires a history of gradually declining functional ability and delusional symptoms absent of a full mood syndrome. An accurate diagnosis requires following a patient over time and obtaining a complete history to partition out the mix of psychotic symptoms and mood disturbance. Clinicians might also assign patients the diagnosis of schizoaffective disorder, a debatable middle diagnostic ground [1]. Although the cross-sectional symptoms may be similar, pharmacological management and prognostic information for bipolar disorder and schizophrenia are different. Second-generation 
atypical medications are prescribed to treat both disorders, but their use for bipolar patients is often intermittent and for shorter duration. Mood stabilizers, the front line treatment course for bipolar disorder, are generally ineffective for schizophrenia. Other treatment modalities and clinical decisions also depend upon the particular SMI condition. Thus, an inappropriate diagnosis may affect treatment decisions and suggest an inaccurate prognosis.

Intertwined with the complexity of distinguishing between these conditions is the question of diagnostic stability. One hundred years ago, Kraepelin asserted that schizophrenia and affective disorders were clearly distinct psychiatric conditions [2]. The debate remains intriguing, with some investigators supporting a psychosis - affective dichotomy [3,4]. Yet, despite codification inherent in the Diagnostic and Statistical Manual for Mental Disorders (DSM), others argue for the existence of a continuum $[5,6]$ between these conditions. A substantial portion of SMI patients receive a diagnosis of both schizophrenia or bipolar disorder, even within a short period. Race or ethnicity may be associated with the stability of SMI diagnosis since distinct longitudinal patterns have been observed across ethnic groups. For example, Chen reported that African Americans change more often from bipolar to schizophrenia diagnoses over time than white patients, and less frequently in the opposite direction [7].

Schizophrenia is generally considered to be the more serious and debilitating disorder [8]. The social stigma acquired through a schizophrenia label may contribute to a lower quality of life $[9,10]$, and prove detrimental to prognosis $[11,12]$. Due to these concerns, the phenomenon of diagnosing schizophrenia rather than bipolar disorder has been labeled "misdiagnosis in a downward direction" [13].

Unfortunately, factors other than clinical presentation and history may affect diagnosis. Past studies indicate that race/ethnicity is one such factor. From the 1970s onward, differential diagnostic patterns have been found consistently in both small observational studies and in larger survey projects [14-21], with minorities being diagnosed with schizophrenia at considerably higher rates than white patients. Despite this composite picture, these studies varied in sample size, definitions, methods, and attempts to control for SES. As Neighbors points out, while the literature supports an assertion of ongoing diagnostic disparities, the evidence is "neither clear nor definitive" [21].

Despite these repeated reports, little evidence exists of true ethnic differences in the two disorders. When diagnoses are obtained using structured research interviews, both schizophrenia and bipolar disorder have prevalence rates of approximately $0.8-1 \%$ across all ethnic groups. Community studies, such as the Epidemiological Catchment Area (ECA) study and National Comorbidity Survey (NCS), found no prevalence differences by ethnicity for either condition after controlling for socioeconomic status (SES) [22, 23].
SES is a significant consideration in any discussion regarding psychiatric diagnosis. As early as 1854, Jarvis documented a relationship between "insanity" and lower social class [24]. Recent studies have confirmed the contribution of disadvantageous SES towards schizophrenia: this disorder has been estimated at up to $8 \times$ more prevalent in lower versus higher SES groups [25-27]. Significant associations have also been shown between lower educational levels [23], lower occupational status [28], and urban residency [29]. SES level has also been shown to be highly correlated with prognosis and outcomes [30]. However, while SES is a potentially important confounding factor, it seems to manifest itself less variably within a veteran population. Most veterans using VA services, especially SMI individuals, tend to occupy the lower end of the SES spectrum [31]. SES has been shown to contribute little towards care-seeking behavior or outcomes among veterans using VA services $[32,33]$.

Aside from SES, reasons for the differential diagnosis are unclear. Numerous theories have been suggested. Most frequently cited is a potential lack of provider cultural sensitivity when interpreting patient symptoms [34-38]. As Rogler notes, "the cultural distance between the diagnostician and the client affects the degree of psychopathology inferred and affects, too, the type of disorder diagnosed" [39]. Specific examples include linguistic differences or culturally specific vernacular being mistakenly identified as a thought disorder, a perception of hostile attitudes and elevated dangerousness, "excessive" somatic complaints, defensive body language, or expressions of "healthy paranoia" by minority patients being seen as pathological $[20,40]$. Even the content and amount of clinical information solicited during a patient encounter has been shown to vary depending on ethnicity [41]. Cultural gaps in the therapeutic process may translate into poor rapport between client and provider, hampering open dialogue and information necessary for accurate diagnosis [42].

Another explanation for divergent diagnostic rates can be found in the differences in expressed symptomatology. Minorities have been reported to display more first rank or positive symptoms on standardized assessment instruments, such as increased frequency and severity of hallucinations, anger, delusions, impulsivity, paranoia or asocial feelings, and expressions of external control. These symptoms may prompt a schizophrenia diagnosis upon first contact with the patient $[20,43,44]$. Minority patients may also experience delays in receiving mental health treatment, either due to access problems or care-seeking behaviors, possibly exacerbating their condition [45-49]. Roukema et al. speculate that delays in seeking treatment factor directly into deteriorating bipolar symptoms, causing this disorder to be mistaken for schizophrenia [50].

Finally, perhaps the most complex interaction is between SMI diagnosis and comorbid substance abuse. Per the ECA, $47 \%$ of patients with schizophrenia also have a diagnosis of either alcohol or drug abuse. Concurrent 
substance abuse may alter psychiatric symptom presentation and subsequent clinical diagnosis. Cannabis in particular, but also alcohol and cocaine, can exacerbate hallucinations, paranoia, mood swings, and violent behavior [51,52]. Some investigators believe substance abuse to be responsible for much of the misdiagnosis problem, regardless of patient ethnicity $[53,54]$.

The NCS found that minorities have lower rates of overall substance abuse than whites (OR for blacks is 0.35 , and 0.80 for Hispanics), although there are differences in alcohol versus drug use, with minorities being less likely to use alcohol, but more likely to misuse other drugs. However, as both Herd and Dawson note, substance abuse imparts greater deleterious effects upon minorities, largely due to lower SES conditions and issues with healthcare access $[55,56]$. The differential rates in substance use across ethnic groups narrows with age [57]. Potential disparities arise here as well, since minorities presenting with dual diagnoses tend to be evaluated and treated for only one condition more often than white patients [58].

The VA has successfully reduced ethnic disparities in diagnosis and treatment across a spectrum of other medical conditions [59]. However, the issue of diagnostic patterns within a veteran SMI population has not been thoroughly addressed. In this study, we benefit from a large, comprehensive national sample from the Department of Veterans Affairs Healthcare System to examine whether differential psychiatric diagnosis is still present in the late 1990s. Increased sensitivity to the issue of cultural competency, plus a recent Surgeon General's report focusing on ethnicity and mental health concerns [60], confirms the timely significance of this research area.

The VA serves a unique mission and is devoted to a special patient population. It is a highly integrated system, in terms of clinical processes and data availability. The largest healthcare organization in the United States, the VA provided services to over 4 million veterans in 2002. The VA is often considered a rather "closed" healthcare system, treating individuals with relatively few other options for care. The length of patient tenure within the system, plus their satisfaction [61] and sense of connection in regard to VA services is generally very high. This combination of a captured and dedicated patient population, coupled with a notably low general SES level, makes the VA a "natural laboratory" for examining this issue. This study is by far the largest investigation of ethnic disparities in the diagnosis of SMI patients. Furthermore, the size and comprehensive nature of the data available through the National Psychosis Registry allows us to adjust for many potentially confounding factors.

The objective of this study examines whether ethnic minority patients were diagnosed with schizophrenia rather than bipolar disorder more frequently than whites, controlling for SES and other patient characteristics. We hypothesize that despite attention devoted to minimizing ethnic disparities, plus the low SES variability and lack of financial barriers to access, minority vet- erans will nevertheless have a greater likelihood of receiving a schizophrenia diagnosis.

\section{Methods and data analysis}

\section{Data source}

Data on patient demographics, diagnoses, and healthcare utilization were obtained from the National Psychosis Registry [62]. The Registry was developed by the Serious Mental Illness Treatment Research and Evaluation Center (SMITREC) to monitor care provided to SMI veterans. It is a comprehensive database and annual report covering all veterans with a serious mental illness (SMI), and includes information on demographics and other patient characteristics, inpatient and outpatient utilization, pharmacy use, and costs. All patients receiving a diagnosis of a schizophrenia, schizoaffective disorder, or bipolar disorder between October 1, 1998 and September 30, 1999 were included in the study population. Veterans were classified into only one of the three major diagnoses; if patients received more than one primary diagnosis during this period, an algorithm based upon the most frequent category was used. This resulted in a total population of 134,523 veterans with a qualifying diagnosis.

For a secondary analysis examining the role of symptom presentation and severity, supplemental data were also used from another veteran SMI population. The Long-Term Mental Health (LTMH) project dataset is a subset of the Registry, with approximately $95 \%$ of individuals interviewed for LTMH also included in the Registry. For this subanalysis, Brief Psychiatric Rating Scale (BPRS) scores from the LTMH questionnaires provided detailed information regarding clinical symptomatology for 1,635 veterans with either schizophrenia or bipolar disorder. Recognizing the importance of clinical presentation during the diagnostic process, this secondary analysis reveals essential information regarding the association between symptom severity and the diagnosis of psychiatric illness.

\section{Measures}

Ethnicity was categorized into three groups for analytical purposes: White, African American or Hispanic. Much effort was devoted in creating the Registry to minimize missing race data, and valid information was obtained for approximately $90 \%$. A variety of patient level factors were incorporated into the analytic models to reflect demographic and illness severity characteristics pertinent to a diagnosis of schizophrenia. Demographic variables included gender, marital status, age, and mortality during the year. Urban residency reflected whether or not a patient lived within a defined metropolitan statistical area. Utilization data within the past year served as a proxy indicator for severity of illness, with specific measures being the number of hospitalization days and outpatient mental health specialty visits. Albeit not a perfect surrogate for illness severity, it is conceptually valid, and a consistent association with utilization has been demonstrated in both medical and mental health treatment [63-65]. A utilization measure also captures the frequency of psychiatric specialty contacts, and correlates with opportunities to receive an SMI diagnosis.

Two other relevant patient characteristics were also examined to determine their relationship with SMI diagnosis. Reflecting the percentage of a patient's disability attributable to his or her military commitment, service connection functions as another proxy for illness severity, in addition to representing a measure of access to and reliance upon VA care. A close association between this variable and health services utilization has been demonstrated [66]. A "tenure" variable has also been created to describe the number of years each patient has been cared for by VA providers in the past decade, and denotes a measure of treatment longevity within the system. As with the number of mental visits, we assumed that tenure level might be associated with accuracy or consistency of diagnosis and, therefore, wished to control for this factor.

Evaluating the role of comorbid substance abuse is complex as 
previously discussed. In lieu of longitudinal and other data pertaining to the relationship between substance abuse and psychosis, a simple control variable was created. This dichotomous measure reflects the existence of an additional outpatient diagnosis for either alcohol or other substance use, dependence or abuse. For the purposes of this study, two factors relating to substance abuse are pertinent: possible differences in dual diagnosis rates by ethnicity, and whether or not increased symptom severity is observed.

The National Psychosis Registry confirmed the low variability of socioeconomic status among SMI veterans: $95 \%$ of these individuals reported annual incomes below $\$ 26,000$. This variable was not included in the primary model since approximately $60 \%$ of patients did not report income information. Yet, given the potential significance of this factor, we endeavored to study its association with schizophrenia. A further exploratory investigation was conducted in a sub-analysis to gauge the impact of low SES.

\section{Data Analysis}

Controlling for potentially confounding variables as previously described, a multinomial logistic regression model was used to estimate the increased probability of being diagnosed with schizophrenia (psychosis) versus bipolar disorder (affective) by race. This analysis calculated the odds ratio (OR) of all factors contributing to a diagnosis of schizophrenia, including the likelihood of an ethnic minority receiving that diagnosis in comparison with a white patient. This model also provided a separate OR for schizoaffective versus bipolar disorder, and explored the theoretical aspects of a 'continuum' in schizophrenia-affective diagnoses. Finally, separate analyses using a LTMH dataset were conducted to incorporate clinical symptomatology and the role of actual illness presentation on psychiatric diagnosis. Statistical analyses were completed using $\mathrm{SAS}^{\circledR}$ System for Windows, Release 8.02 by SAS Institute Inc., Cary, N. C.

\section{Results}

Of the entire population, $53.1 \%(71,363)$ had an inclusion diagnosis of schizophrenia; another $36.9 \%(48,443)$ were classified as bipolar, with the remaining $10.9 \%$ $(14,717)$ receiving a schizoaffective diagnosis. The ethnic composition was $69.5 \%$ White, $23.6 \%$ African American, and $6.9 \%$ Hispanic. Characteristic of an SMI population, less than $30 \%$ of these patients are married, their average age was 52.6 years, and they are high utilizers of care. The mean number of outpatient mental health visits was 28.1 (s.d.=54.1), with an average number of inpatient days of $13.6($ s. d. $=36.0)$. The mean income for this sample was only $\$ 11,845$ annually (s.d. $=\$ 8,344$ ). Regarding the stability of diagnosis, it should be also noted that over $25 \%$ of all patients received more than one category of the SMI diagnoses in the same year. Table 1 presents the descriptive statistics for this patient group, broken down by both diagnoses and ethnicity. As a Registry subset, the LTMH profile is very similar to the parent dataset in terms of patient characteristics and utilization measures. For example, the mean inpatient days and outpatient mental health visits were 14.1 and 30.4 for LTMH patients compared to 13.6 and 28.1 for the Registry.

\section{Bivariate results}

Table 1 presents a comparison of the key model measures by diagnosis and ethnic group. All of the bivariate associations between ethnicity or diagnosis and the other variables were statistically significant $(\mathrm{p}<0.05)$. Regarding ethnicity, greater differences are found between the two minority groups themselves than between themselves and White patients. African Americans and Hispanics contrasted sharply with each other on rates of urban residency, gender, dual diagnosis, marital status and utilization. In terms of anticipated risk factors for schizophrenia, Hispanics actually displayed a slightly more favorable profile than either of the other ethnic groups. Compared with White patients, both minority groups had better access in terms of service connection and demonstrated longer "tenure" within the VA system. Several other variables characterized essential differences pertinent to this study. Overall, patients with schizophrenia are much more likely to be male, unmarried, utilize greater services, report lower income, and have been treated within the VA system longer. Comorbid substance abuse, however, was considerably higher in bipolar patients.

\section{Multivariate results}

Patients who were male, unmarried, or living in a rural environment were more likely to be diagnosed with schizophrenia rather than bipolar disorder. In addition, higher mortality and service connection was also strongly associated with a schizophrenia diagnosis, as was longer tenure within the VA care system. Although statistically significant, minimal or no effects were noticed for age, and either of the utilization variables. Recognizing the predominantly male VA population, an interaction model with gender ${ }^{\star}$ diagnosis was also run, with no significant findings. Table 2 provides the adjusted odds ratios for each specific factor.

An appreciably different picture emerged when considering the question of ethnicity. After controlling for possible confounders, race continued to most strongly predict a schizophrenia diagnosis. The odds ratio for African Americans was 4.05 (95\% C. I. 3.91-4.19), indicating over four times greater likelihood of being diagnosed with schizophrenia instead of bipolar disorder relative to white patients. For Hispanics, the risk was also highly elevated, with an OR of 3.15 (95\% C. I. 2.97-3.33).

Patients with comorbid substance abuse were more likely to be diagnosed with bipolar disorder. Interaction models exploring the link between ethnicity ${ }^{\star}$ dual diagnosis and schizophrenia did not provide additional insights or alter the primary findings of this study. That is, African American or Hispanic patients with a substance abuse disorder were no more likely to be given a schizophrenia diagnosis than those minorities without this comorbidity.

Turning to the second perspective examining schizoaffective versus bipolar diagnoses, we found sim- 
Table 1 Descriptive results

\begin{tabular}{|c|c|c|c|c|}
\hline & All & Schizophrenia & SchizoAffective & Bipolar \\
\hline $\mathrm{N}_{\%}$ & 134,523 & $\begin{array}{l}71,363 \\
53.1 \%\end{array}$ & $\begin{array}{l}14,717 \\
10.9 \%\end{array}$ & $\begin{array}{l}48,443 \\
36.9 \%\end{array}$ \\
\hline $\begin{array}{l}\text { Age (mean) } \\
\text { (SD) }\end{array}$ & $\begin{array}{r}52.6 \\
(12.7)\end{array}$ & $\begin{array}{r}53.9 \\
(12.8)\end{array}$ & $\begin{array}{r}50.2 \\
(11.3)\end{array}$ & $\begin{array}{r}51.5 \\
(12.7)\end{array}$ \\
\hline \multicolumn{5}{|l|}{ Ethnicity } \\
\hline White & $69.5 \%$ & $59.9 \%$ & $71.6 \%$ & $83.1 \%$ \\
\hline African American & $23.6 \%$ & $31.2 \%$ & $22.4 \%$ & $12.8 \%$ \\
\hline Hispanic & $6.9 \%$ & $8.9 \%$ & $6 \%$ & $4.1 \%$ \\
\hline Female, $\%$ & $6.7 \%$ & $4 \%$ & $8.6 \%$ & $10.1 \%$ \\
\hline Married, \% & $29.9 \%$ & $25.8 \%$ & $27.9 \%$ & $36.4 \%$ \\
\hline Died in FY99, \% & $2.3 \%$ & $2.8 \%$ & $1.6 \%$ & $1.6 \%$ \\
\hline MSA resident, $\%$ & $79.1 \%$ & $78.2 \%$ & $81.5 \%$ & $79.6 \%$ \\
\hline Dual dx, \% & $30 \%$ & $25.6 \%$ & $32.6 \%$ & $35.7 \%$ \\
\hline Service connection \% & 67.1 & 74.6 & 68.2 & 53.3 \\
\hline $\begin{array}{l}\text { Institutional days } \\
\text { (SD) }\end{array}$ & $\begin{array}{r}13.6 \\
(36.0)\end{array}$ & $\begin{array}{r}14.9 \\
(42.7)\end{array}$ & $\begin{array}{r}14.5 \\
(39.5)\end{array}$ & $\begin{array}{r}11.3 \\
(26.1)\end{array}$ \\
\hline $\begin{array}{l}\text { Number of MH stops } \\
\text { (SD) }\end{array}$ & $\begin{array}{r}28.1 \\
(54.1)\end{array}$ & $\begin{array}{r}28.8 \\
(64.0)\end{array}$ & $\begin{array}{r}32.2 \\
(60.7)\end{array}$ & $\begin{array}{r}25.8 \\
(48.2)\end{array}$ \\
\hline $\begin{array}{l}\text { Tenure (past } 10 \text { years) } \\
\text { (SD) }\end{array}$ & $\begin{array}{r}8.2 \\
(2.9)\end{array}$ & $\begin{array}{r}8.7 \\
(2.5)\end{array}$ & $\begin{array}{r}8.2 \\
(2.8)\end{array}$ & $\begin{array}{r}7.3 \\
(3.2)\end{array}$ \\
\hline \multirow[t]{2}{*}{$\begin{array}{l}\text { Income } \\
\text { (SD) }\end{array}$} & $\begin{array}{l}\$ 11,845 \\
(\$ 8,344)\end{array}$ & $\begin{array}{l}\$ 10,358 \\
(\$ 7,343)\end{array}$ & $\begin{array}{l}\$ 10,966 \\
(\$ 7,821)\end{array}$ & $\begin{array}{r}\$ 13,294 \\
(\$ 11,887)\end{array}$ \\
\hline & & White & African-American & Hispanic \\
\hline $\mathrm{N}$ & & 93,507 & 31,756 & 48,443 \\
\hline $\begin{array}{l}\text { Age (mean) } \\
\text { (SD) }\end{array}$ & & $\begin{array}{r}53.7 \\
(13.0)\end{array}$ & $\begin{array}{r}49.3 \\
(11.1)\end{array}$ & $\begin{array}{r}53.5 \\
(12.6)\end{array}$ \\
\hline Female, $\%$ & & $6.9 \%$ & $7.1 \%$ & $3.3 \%$ \\
\hline Married, \% & & $30.2 \%$ & $22.7 \%$ & $50.9 \%$ \\
\hline Died in FY99, \% & & $2.5 \%$ & $1.8 \%$ & $1.7 \%$ \\
\hline MSA resident, $\%$ & & $77.8 \%$ & $89.1 \%$ & $57.4 \%$ \\
\hline Dual dx, \% & & $26.7 \%$ & $41.1 \%$ & $24.9 \%$ \\
\hline Service connection \% & & 65.9 & 68.0 & 74.2 \\
\hline $\begin{array}{l}\text { Institutional days } \\
\text { (SD) }\end{array}$ & & $\begin{array}{r}14.0 \\
(47.2)\end{array}$ & $\begin{array}{r}14.1 \\
(41.6)\end{array}$ & $\begin{array}{r}7.5 \\
(30.4)\end{array}$ \\
\hline $\begin{array}{l}\text { Number of MH stops } \\
\text { (SD) }\end{array}$ & & $\begin{array}{r}27.3 \\
(58.1)\end{array}$ & $\begin{array}{r}32.5 \\
(62.3)\end{array}$ & $\begin{array}{r}20.9 \\
(47.8)\end{array}$ \\
\hline $\begin{array}{l}\text { Tenure (past } 10 \text { years) } \\
\text { (SD) }\end{array}$ & & $\begin{array}{r}8.0 \\
(3.0)\end{array}$ & $\begin{array}{r}8.5 \\
(2.6)\end{array}$ & $\begin{array}{r}8.6 \\
(2.6)\end{array}$ \\
\hline $\begin{array}{l}\text { Income } \\
\text { (SD) }\end{array}$ & & $\begin{array}{l}\$ 12,818 \\
(\$ 9,655)\end{array}$ & $\begin{array}{r}\$ 8,915 \\
(\$ 6,764)\end{array}$ & $\begin{array}{l}\$ 11,084 \\
(\$ 9,201)\end{array}$ \\
\hline
\end{tabular}

ilar but less dramatic results. Minimal differences in effect sizes can be seen for patient characteristics and utilization. Ethnicity, once again, is clearly the prevailing factor: the OR for African Americans and Hispanics here was 1.95 and 1.69 , respectively. Although lower in comparison to a schizophrenia diagnosis, these remain highly elevated and significantly increased probabilities relative to White patients.

\section{Secondary analyses}

\section{SES/income}

A separate analysis was performed on those individuals that possessed valid income data for FY99 $(\mathrm{N}=49,901)$, and the logistic regression re-run for a schizophrenia versus bipolar diagnosis. After viewing the overall distribution, income was included as a categorical variable $(<\$ 10 \mathrm{~K}, \$ 10-20 \mathrm{~K}, \$ 20-30 \mathrm{~K}, \$ 30+)$. The first notable finding was a direct linear relationship with psychosis: confirming expectations, as income dropped, the probability of a schizophrenia diagnosis increased (OR for highest SES was 0.51 compared to lowest). Secondly, and most significantly, when income was first added then dropped from the model, the risk of a schizophrenia diagnosis in African Americans and Hispanics was only attenuated $3 \%$ and $4 \%$, respectively. While not minimizing the conceptual importance of SES factors, these results help support our hypothesis that it plays a rather minor role within a SMI veteran population. Evidence of differential diagnosis patterns across ethnic groups was certainly not eliminated when including income in this study. 
Table 2 Multivariate results

\begin{tabular}{llllll}
\hline & \multicolumn{2}{l}{ SCH vs. BPa } & & \multicolumn{2}{l}{ SCH vs. SchAffa } \\
\cline { 2 - 3 } \cline { 6 - 6 } Variable & Odds Ratio & $95 \% \mathrm{Cl}$ & & Odds Ratio & $95 \% \mathrm{Cl}$ \\
\hline Age & 1.01 & $1.01-1.01$ & & 0.99 & $0.99-0.99$ \\
Female & 0.35 & $0.34-0.38$ & & 0.71 & $0.67-0.77$ \\
Married & 0.47 & $0.46-0.49$ & & 0.66 & $0.63-0.69$ \\
OP mental health stops & 1.00 & $1.00-1.00$ & & 1.00 & $1.00-1.00$ \\
Institutional days & 1.00 & $1.00-1.00$ & & 1.00 & $1.00-1.00$ \\
Mortality (died in FY99) & 1.71 & $1.56-1.87$ & & 1.10 & $0.95-1.28$ \\
MSA resident & 0.85 & $0.83-0.88$ & & 1.02 & $0.97-1.07$ \\
Tenure in VA system & 1.11 & $1.11-1.12$ & & 1.09 & $1.08-1.10$ \\
Substance abuse/dual dx & 0.52 & $0.50-0.53$ & & 0.68 & $0.65-0.71$ \\
Service connection: & & & & \\
(reference is non-connected) & & & & \\
$\quad 0-49$ & 0.79 & $0.76-0.82$ & & 0.77 & $0.73-0.81$ \\
$\quad$ 50-69 & 0.99 & $0.94-1.05$ & & 0.86 & $0.79-0.94$ \\
$\quad$ 70+ & 2.27 & $2.20-2.34$ & & 1.65 & $1.58-1.73$ \\
Race: & & & & \\
$\quad$ Black vs. White & 4.05 & $3.91-4.19$ & & 1.95 & $1.86-2.05$ \\
$\quad$ Hispanic vs. White & 3.15 & $2.98-3.33$ & & 1.69 & $1.56-1.84$ \\
\hline
\end{tabular}

a SCH schizophrenia; $B P$ bipolar disorder; SchAff schizoaffective disorder

\section{Presentation of symptoms (LTMH data)}

Recognizing a multitude of possible confounding factors pertaining to care-seeking behavior, the use of nonVA providers and services, plus provider and system level factors that could not be addressed through an administrative database, we examined the role of symptom presentation in the clinical encounter. If indeed minority veterans presented with more severe symptoms, then such differential symptomatology might explain our diagnostic findings. These differences could be attributed to substance abuse, access problems or health beliefs delaying care until their psychiatric condition deteriorated.

Although the Psychosis Registry does not contain information regarding symptoms or severity of condition, data on a similar population were observed in the second large VA dataset. The LTMH project provides BPRS scores of 1,635 veterans with schizophrenia and bipolar disorder. For the purposes of this study, a bivariate comparison of positive and negative symptoms across ethnic groups was conducted. Only minor differences were observed in the individual symptoms; in fact, when dichotomized into moderate or worse severity (score of $3+$ on any item, 0-6 scale), a level commonly viewed as a key diagnostic predictor, none were significantly different by ethnicity. Moreover, of the three symptoms where minority veterans did have higher mean scores, two of these (elation and grandiosity) are typically indicative of a bipolar disorder more than schizophrenia.

No differences in symptomatology were found between patients with comorbid substance abuse compared to those without a dual diagnosis, regardless of ethnicity. Interestingly, for both the Registry and LTMH populations, mean GAF scores were also slightly higher in African Americans; this implies a higher functioning patient group as perceived by providers, a description contrary to the common perception of individuals with schizophrenia. Per the Registry, the average GAF scores for veterans with schizophrenia was 49.2 versus 54.1 for bipolar patients. As a final analysis, equivalent logistic models previously run on the Registry data were duplicated in the LTMH sample; the elevated odds ratios placing minorities with a great probability for a schizophrenia diagnosis were only attenuated about $5 \%$ when presenting symptoms were incorporated. These results provide some confirmatory evidence of diagnostic disparities regardless of SMI symptoms or severity of illness (Figs. 1 and 2).

\section{Discussion}

To our knowledge, this is the first study to explore the topic of potential misdiagnosis by race within a large national healthcare organization. Despite improvements made in numerous other medical areas, these findings confirm evidence of lingering disparities in psychiatric diagnosis across ethnic groups. Consistent with previous research, a higher percentage of minority patients received a diagnosis of schizophrenia. With the exception of a moderately protective effect for urban living, the results confirmed and expanded upon previous findings in the literature. Specifically, race appears to matter and still appears to adversely pervade the clinical encounter, whether consciously or otherwise. The highly elevated likelihood of a minority veteran being classified as psychotic endured when controlling for a constellation of relevant patient factors. Interestingly, despite exhibiting a generally protective profile as compared to White patients, the greater probability of schizophrenia for Hispanic veterans appears especially pronounced.

An elevated but attenuated likelihood for schizoaf- 


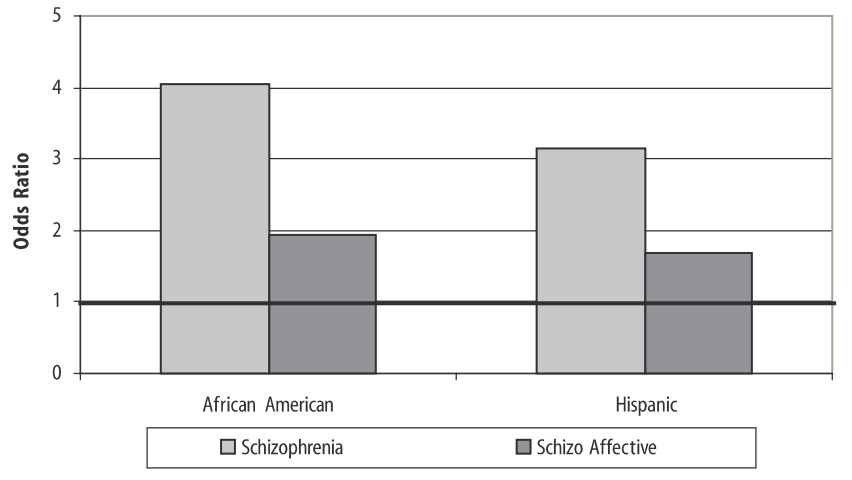

Fig. 1 Risk of schizophrenia and schizoaffective diagnoses by ethnic group

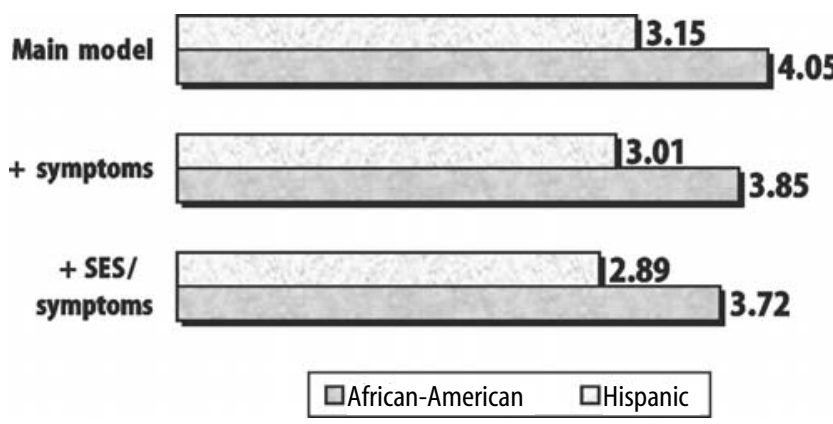

Fig. 2 Risk of schizophrenia diagnosis. Main model from primary Psychosis Registry dataset; attenuated OR for influence of symptoms and SES from LTMH and Registry data, respectively

fective disorder in comparison with schizophrenia can be interpreted as lending some credence to the continuum theory. That is, as a patient moves downwards towards a more serious SMI condition, the probability of an ethnic minority receiving that diagnosis increases accordingly. Also, as previously noted, a sizable proportion of Psychosis Registry patients received more than one primary diagnosis in FY99. Similar to the findings of Chen, this also had an ethnic component, as minorities were more likely to fluctuate between psychotic and affective conditions. Restricting the analysis to individuals with only one diagnosis might shed additional insight into the clinical encounter, as would a longitudinal examination of the same patients over time.

The two conceivable areas of most significance for potential confounding were socioeconomic status and comorbid substance abuse. Both of these variables could certainly affect symptom presentation or the manifestation of psychosis and, therefore, how a provider assessed a patient. However, the fact that each of these variables only marginally contributed to an elevated schizophrenia risk (i. e., 3-5\% each) makes it unlikely they are adversely influencing our results.

Potentially more problematic is the question of comorbid substance abuse. In addition to the complex and unexplored causal associations, plus detrimental effects on care-seeking behavior, the presence of a dual diagnosis can exacerbate psychotic symptoms. If so, an assignment of schizophrenia becomes quite reasonable and differential diagnosis rates might be at least partially explained by different substance abuse prevalence across ethnic groups. However, although African Americans had a much higher rate of dual diagnosis than Whites, Hispanic patients actually had a lower rate. Secondly, no effects were observed in the relationship between substance abuse and symptom severity, nor with the interaction between schizophrenia, ethnicity and dual diagnosis status. This finding echoes the work of Munley et al. who reported no differences in symptomatology between African American and White veterans with both schizophrenia and substance abuse [67]. The seemingly "protective" effect for being diagnosed with schizophrenia is misleading and simply in line with a substantial literature noting higher rates of comorbidity among bipolar patients [68-70].

\section{Limitations}

Despite considerable attention to relevant confounding factors, this is foremost a descriptive and retrospective study using administrative data. Thus, although we are able to document strong evidence regarding diagnostic disparities, we cannot directly address specific reasons for this issue. Notwithstanding an exploratory analysis, the study could also not fully examine the role of symptom presentation, nor incorporate provider clinical experience, judgment or interpretation of psychotic elements. Other dynamics might also affect these findings, such as provider ethnicity and professional licensure (e.g., psychiatrist, psychologist, MSW, mental health specialist versus primary care, etc.), provider training and cultural norms of practice, geographic region of the country and/or ethnic composition of the area, and whether or not the VA medical center has an academic affiliation. Another unique feature of the VA, a predominantly male population, should also be noted again. While this might somewhat limit generalization to other systems, we found no evidence suggesting in the literature or our analysis a differential effect between gender and ethnicity.

We did not model the presence of post-traumatic stress disorder (PTSD), which could also complicate the diagnostic process due to overlapping symptomatology with psychosis. However, the current literature is somewhat mixed regarding prevalence rates, epidemiology, and differential impact of PTSD symptomatology [71, 72]. In some studies, Hispanics have higher PTSD rates than other groups [73], though African Americans might have more sub-clinical psychotic symptoms [74]. Yet other VA researchers have found no PTSD prevalence or symptom differences by race $[75,76]$. Furthermore, Frueh (2002) did not find differences in the diagnosis of psychosis in PTSD patients, despite these higher PTSD symptoms levels.

Care-seeking behavior and access delays raise another interesting and pertinent point, as we were unable to specifically model to what extent these issues affect 
SMI diagnosis. Disparities have been noted in mental health treatment and utilization rates. For example, minorities have been shown to receive less specialty mental health services [77], and fewer atypical antipsychotics [78]. Given the mandate to provide care to all veterans, problems with access by ethnicity vary little within the VA. Harada has investigated the role of racial identity and satisfaction on willingness to seek healthcare, and differences between ethnic groups [79, 80], with no additional burden placed upon minorities. Since veterans seeking care within the VA tend to be sicker than those receiving treatment outside the system [81], minimal bias from caring for only the healthiest individuals is also minimized here. Yet, if these factors contribute to greater illness severity upon reaching a provider, then a diagnosis of schizophrenia could be more likely regardless of ethnicity.

Similarly, this study does not address how patients seek care outside of the VA, nor to the extent that possible selection factor issues result. However, several studies have demonstrated that ethnic minorities might be more comfortable and willing to seek mental healthcare services $[82,83]$, and also tend to utilize such services within the VA more than White veterans [84]. Access difficulties have also been shown to be rather minimal, with no differences by ethnicity; Washington and colleagues concluded that the VA's ambulatory care system is effective in mitigating ethnic disparities in this regard [85]. In addition, Hoff and Rosenheck note that veterans with either schizophrenia or high service connection have minimal "out of system" use [86]. Given this background and the fact that illness severity was equivalent across ethnic groups, we are confident that access issues or selection bias are unlikely to account for our findings.

We recognize that income might be viewed as a poor proxy for SES, and that the latter is not synonymous with social class, occupational status or income alone. This debate has led researchers to rely upon a combination of measures to capture SES, with no clear consensus of the most appropriate method. The complex association with psychosis should certainly not be dismissed lightly as SES has multiple dimensions. For example, we note that occupation, but interestingly not education, has been shown to play a role in the diagnosis and prognosis of schizophrenia [30]. Ideally, a finer multifaceted form of SES could be incorporated analytically to study this issue. However, given the overall lower SES status for SMI veterans across many dimensions, we again believe this is a relatively minor factor within the VA in terms of diagnostic influence.

From a sociological perspective, there is also the practical question of how "race" itself is classified and used in administrative database. Prior and subsequent to the 2000 census, the literature has focused attention primarily upon the complicated and confounding nature of using ethnicity in health services research [87-89]. Although by no means a trivial issue, this complex subject falls outside the scope of our analysis. Accuracy or extensive missing race data in VA administra- tive records can be a potential problem as well. Fortunately, a recent article supports internal VA discussion and analysis confirming the accuracy of veteran ethnicity data, and the appropriateness of relying on that information in this study [90].

Despite these possible limitations, we return once more to actual symptom presentation. The fact that overt illness severity did not appear to vary by race irrespective of the influence of SES, comorbid substance abuse, and possible care-seeking differences lends some credence to the term "diagnostic disparity". In a smaller observational study, Hutchinson also determined that minor differences in symptomatology cannot account for variations in schizophrenia diagnosis [91]. Furthermore, in separate research efforts, Weisman and Brekke found that ethnic minorities displayed significantly fewer psychotic symptoms than White patients $[92,93]$. Clearly, the relevance of psychotic symptoms to clinical diagnosis highlights these findings and suggests that additional work in this area is required.

Notwithstanding numerous challenges to psychiatric classification itself, from Thomas Szasz to more recent critics [94], many forces shape the clinical encounter and subsequent diagnosis. The powerful influence of social construction in mental health [95] has also been noted, in addition to the questionable usefulness of a schizophrenia label itself [96]. The combination of patient, provider and system factors, plus other unrecognized dimensions, conspires to produce an extremely difficult and daunting assessment task for the clinician. Choosing one diagnosis over another is indeed a complex process. As Westermeyer nicely summarizes, "diagnosis involves more than a mere Linnaean classification of homogeneous entities" [97]. Researchers and clinicians alike can appreciate why diagnostic stability and accuracy debates have lingered for such a long time.

Several interesting policy implications arise from these findings. Foremost is the role of DSM criteria in clinical practice and some have questioned how frequently it is truly consulted in clinical practice [98]. Jones and others have commented that the DSM-III was specifically intended to introduce greater standardization in SMI diagnoses, while reducing the ethnic component [40]. Yet, conversely, the DSM-IV is commonly believed to represent a tremendous advancement by more specifically incorporating culture into the clinical process [99]. In addition to doubting the success of either revision, the importance of cultural factors raises the debate over the very appropriateness of this mission, that is, should culture be overtly excluded from diagnostic assessment? In either case, the use of culturally sensitive structured instruments (e. g., CIDI) has also been advocated to minimize diagnostic variability [100].

As mentioned, organizations should also recognize the contribution of provider and system factors, and not only patient dynamics. In that light, ethnic matching of patient and provider is occasionally forwarded as a possible remedy. However, previous research has shown negligible results in terms of mental health outcomes, or 
reducing the imbalance of SMI diagnosis by ethnicity [101-103]. Above all, regardless of other considerations, the overwhelming body of literature and results clearly encourages the promotion of greater cultural competency and awareness in working with minority patients. We also note that calls for improvement in this area are not equivalent to charges of "racial bias", nor do these results regarding diagnostic differences in any way imply such criticism.

In line with the results of this study and the policy implications, there exists a rich avenue for future research directions. Further research might focus upon first-contact appointments and diagnosis (e.g., hospital admission, or initial outpatient visit), limiting the sample to patients with only one primary diagnosis, and a longitudinal analysis to gauge stability over time. With available data, the influence of provider and system factors would also be illuminating, as would an exploration of regional differences: the Psychosis Registry has revealed substantial differences in diagnostic patterns across the VA's 22 national networks or VISNs. Finally, the link between clinical assessment, actual treatment, and outcomes will document the actual role of diagnosis, i. e., how treatment might differ across ethnic groups depending on the assigned SMI diagnosis.

As Zuvekas et al. note in their recent decomposition of contributing factors, a sizable portion of ethnic disparities in any medical realm simply cannot be explained via observable data, no matter how comprehensive [104]. Numerous conceptual themes and practical concerns are involved: cultural context and sensitivity, fine shades of diagnostic meaning, confounding comorbidities, patient-provider rapport, SES factors, role of strict DSM application, health beliefs, care-seeking behavior, access issues, and so on. Nevertheless, in controlling for pertinent patient side factors, our findings not only demonstrate a profound pattern of diagnostic disparities, but also represent a significant step in better delineating the intricate black box of possible causes.

\section{Conclusion}

This study utilized a comprehensive national database to delve into the complex waters of psychiatric diagnosis. Despite the acknowledged limitations of presenting descriptive inferences in psychiatric diagnosis through administrative data, these results highlight an ongoing concern in the evaluation and treatment of SMI veterans. Recognizing the multi-faceted nuances that comprise the mental health encounter, patient factors alone cannot explain the widely divergent diagnostic patterns seen here. The acutely elevated risk of a schizophrenia designation in minority patients warrants serious attention, especially considering the significant ramifications for appropriate treatment and outcomes. Accurate assessment of these vulnerable veterans requires a heightened sensitivity and awareness to cultural competency as the VA continues striving to eliminate ethnic dispari- ties in all spheres of clinical practice. In addition to a more refined examination of symptom presentation and comorbid substance abuse issues, further research should focus upon relevant provider and system factors that contribute to psychiatric diagnosis.

\section{References}

1. American Psychiatric Association. DSM-IV (1994) Diagnostic and Statistical Manual of Mental Disorders, $4^{\text {th }}$ ed. Washington, D. C.: American Psychiatric Association

2. Jablensky A (1997) The 100-year epidemiology of schizophrenia. Schizophr Res 28(2-3):111-125

3. Brockington IF, Kendell RE, Wainwright S, Hillier VF, Walker J (1979) The distinction between the affective psychoses and schizophrenia. Br J Psychiatry 135:243-248

4. Kendell RE, Brockington IF (1980) The identification of disease entities and the relationship between schizophrenic and affective psychoses. Br J Psychiatry 137:324-331

5. Lapierre YD (1994) Schizophrenia and manic-depression: separate illnesses or a continuum? Can J Psychiatry 39(9 Suppl 2): S59-S64

6. Compton WM, Guze SB (1995) The neo-Kraepelinian revolution in psychiatric diagnosis. Eur Arch Psychiatry Clin Neurosci 245 (4-5):196-201

7. Chen YR, Swann AC, Johnson BA (1998) Stability of diagnosis in bipolar disorder. J Nerv Ment Dis 186(1):17-23

8. Murray CJ, Lopez AD (1996) Evidence-based health policy lessons from the Global Burden of Disease Study. Science 274(5288):740-743

9. Pinikahana J, Happell B, Hope J, Keks NA (2002) Quality of life in schizophrenia: a review of the literature from 1995 to 2000. Int J Ment Health Nurs 11(2):103-111

10. Voruganti LN, Awad AG, Oyewumi LK, Cortese L, Zirul S, Dhawan R (2000) Assessing health state utilities in schizophrenia. Pharmacoeconomics 17(3):273-286

11. Link BG, Struening EL, Rahav M, Phelan JC, Nuttbrock L (1997) On stigma and its consequences: evidence from a longitudinal study of men with dual diagnoses of mental illness and substance abuse. J Health Soc Behav 38(2):177-190

12. Witztum E, Margolin J, Bar-On R, Levy A (1995) Stigma, labelling and psychiatric misdiagnosis: origins and outcomes. Med Law 14(7-8):659-669

13. Bamgbose O, Edwards D, Johnson S (1980) The effects of race and social class on clinical judgment. J Clin Psychol 36(2): 605-609

14. Watkins BA, Cowan MA, Davis WE (1975) Differential diagnosis imbalance as a race-related phenomenon. J Clin Psychol 31(2): 267-268

15. Bell CC, Mehta $\mathrm{H}$ (1980) The misdiagnosis of black patients with manic depressive illness. J Natl Med Assoc 72:141-145

16. Mukherjee S, Shukla S, Woodle J, Rosen A, Olarte S (1983) Misdiagnosis of schizophrenia in bipolar patients: a multiethnic comparison. Am J Psychiatry 140(12):1571-1574

17. Pavkov TW, Lewis DA, Lyons IS (1989) Psychiatric diagnosis and racial bias: an empirical investigation. Professional Psychology 20:364-368

18. Flaskerud JH, Hu L-T (1992) Relationship of ethnicity to psychiatric diagnosis. J Nerv Ment Dis 180(5):296-303

19. Adebimpe VR (1994) Race, racism, and epidemiological surveys. Hosp Comm Psychiatry 45(1):27-31

20. Strakowski SM, Flaum M, Amador X, Bracha HS, Pandurangi AK, Robinson D, Tohen M (1996) Racial differences in the diagnosis of psychosis. Schizophr Res 21:17-24

21. Neighbors HW, Trierweiler SJ, Munday C, Thompson EE, Jackson JS, Binion VJ, Gomez J (1999) Psychiatric diagnosis of African Americans: diagnostic divergence in clinician-structured and semistructured interviewing conditions. J Natl Med Assoc 91 (11):601-612 
22. Kessler RC, McGonagle KA, Zhao S, Nelson CB, Hughes M, Eshleman S, Wittchen H-U, Kendler KS (1994) Lifetime and 12month prevalence of DSM-III-R psychiatric disorders in the United States: Results from the National Comorbidity Survey. Arch Gen Psychiatry 51(1):8-19

23. Robins LN, Helzer JE, Weissman MM, Orvaschel H, Gruenberg E, Burke JD Jr, Regier DA (1984) Lifetime prevalence of specific psychiatric disorders in three sites. Arch Gen Psychiatry 41(10):949-958

24. vander Stoep A, Link B (1998) Social class, ethnicity, and mental illness: the importance of being more than earnest. AJPH 88(9):1396-1402

25. Aro S,Aro H, Keskimaki I (1995) Socio-economic mobility among patients with schizophrenia or major affective disorder: A 17-year retrospective follow-up. Br J Psychiatry 166(6):759-767

26. Regier DA, Narrow WE, Rae DS, Manderscheid RW, Locke BZ, Goodwin FK (1993) The de facto US mental and addictive disorders service system. Epidemiologic catchment area prospective 1 -year prevalence rates of disorders and services. Arch Gen Psychiatry 50(2):85-94

27. Zhang AY, Snowden LR (1999) Ethnic characteristics of mental disorders in five US communities. Cultural Diversity and Ethnic Minority Psychology 5(2):134-146

28. Antunes G, Gordon C, Gaitz CM, Scott J (1974). Ethnicity, socioeconomic status, and the etiology of psychological distress. Sociol Soc Res 58(4):360-368

29. Torrey EF, Bowler A (1990) Geographical distribution of insanity in America: Evidence for an urban factor. Schizophr Bull 16(4): 591-604

30. Samele C, van Os J, McKenzie K, Wright A, Gilvarry C, Manley C, Tattan R (2001) Does socioeconomic status predict course and outcome in patients with psychosis? Soc Psychiatry Psychiatr Epidemiology 36(12):573-581

31. Oddone EZ, Petersen LA, Weinberger M, Freedman J, Kressin NR (2002) Contribution of the veterans health administration in understanding racial disparities in access and utilization of health care. Med Care 40(Suppl 1):I3-I13

32. Menke TJ, Rabeneck L, Hartigan PM, Simberkoff MS, Wray NP (2000) Clinical and socioeconomic determinants of health care use among HIV-infected patients in the Department of Veterans Affairs. Inquiry 37(1):61-74

33. Ashton CM, Petersen NJ, Wray NP, Yu HJ (1998) The Veterans Affairs medical care system: hospital and clinic utilization statistics for 1994. Med Care 36(6):793-803

34. Jenkins JH (1998) Diagnostic criteria for schizophrenia and related psychotic disorders: integration and suppression of cultural evidence in DSM-IV. Transcultural Psychiatry 35(3): 357-376

35. Lefley HP (1990) Culture and chronic mental illness. Hosp Community Psychiatry 41(3):277-286

36. Lewis-Fernandez R (1996) Cultural formulation of psychiatric diagnosis. Cult Med Psychiatry 20(2):133-144

37. Weisman AG (1997) Understanding cross-cultural prognostic variability for schizophrenia. Cult Divers Ment Health 3(1):23-35

38. Killian TM, Killian LT (1990) Sociological investigations of mental illness: a review. Hosp Community Psychiatry 41 (8):902-911

39. Rogler LH (1996) Framing research on culture in psychiatric diagnosis: the case of the DSM-IV. Psychiatry 59(2):145-154

40. Jones BE, Gray BA (1986) Problems in diagnosing schizophrenia and affective disorders among blacks. Hosp Community Psychiatry $37(1): 61-65$

41. Strakowski SM, Hawkins JM, Keck PE Jr, McElroy SL, West SA, Bourne ML, Sax KW, Tugrul KC (1997) The effects of race and information variance on disagreement between psychiatric emergency service and research diagnoses in first-episode psychosis. J Clin Psychiatry 58(10):457-463

42. Whaley AL (2001) Cultural mistrust of white mental health clinicians among African Americans with severe mental illness. Am J Orthopsychiatry 71(2):252-256

43. Adebimpe VR, Hedlund JL, Cho DW, Wood JB (1982) Symptomatology of depression in black and white patients. J Natl Med Assoc 74(2):185-190
44. Hamberger KL, Hastings JE (1992) Racial differences on the MCMI in an outpatient clinical sample. J Pers Assess 58(1):90-95

45. Leaf PJ, Bruce ML, Tischler GL, Holzer CE $3^{\text {rd }}$ (1987) The relationship between demographic factors and attitudes toward mental health services. J Community Psychol 15(2):275-284

46. Wells K, Klap R, Koike A, Sherbourne C (2001) Ethnic disparities in unmet need for alcoholism, drug abuse, and mental health care. Am J Psychiatry 158:2027-2032

47. Gallo JJ, Marino S, Ford D, Anthony JC (1995) Filters on the pathway to mental health care, II. Sociodemographic factors. Psychol Med 25(6):1149-1160

48. Neighbors HW, Jackson JS (1984) The use of informal and formal help: four patterns of illness behavior in the black community. Am J Community Psychol 12(6):629-644

49. Sussman LK, Robins LN, Earls F (1987) Treatment-seeking for depression by black and white Americans. Soc Sci Med 24(3): 187-196

50. Roukema R, Fadem B, James B, Rayford F (1984) Bipolar disorder in a low socioeconomic population. J Nerv Ment Dis 172(2): 76-79

51. Caspari D (1999) Cannabis and schizophrenia: results of a follow-up study. Eur Arch Psychiatry Clin Neurosci 249(1):45-49

52. Drake RE, Wallach MA (1989) Substance abuse among the chronic mentally ill. Hosp Community Psychiatry 40(10): 1041-1046

53. Brown ES, Suppes T, Adinoff B, Rajan Thomas N (2001) Drug abuse and bipolar disorder: comorbidity or misdiagnosis? J Affect Disord 65(2):105-115

54. Ineichen B (1991) Schizophrenia in British Afro-Caribbeans: two debates confused? Int J Soc Psychiatry 37(4):227-232

55. Herd D (1994) Predicting drinking problems among black and white men: results from a national survey. J Stud Alcohol 55: 61-71

56. Dawson DA (1998) Beyond black, white and Hispanic: race, ethnic origin and drinking patterns in the United States. J Subst Abuse 10(4):321-339

57. Prigerson HG, Desai RA, Rosenheck RA (2001) Older adult patients with both psychiatric and substance abuse disorders: prevalence and health service use. Psychiatr Q 72(1):1-18

58. Horton J, Compton W, Cottler LB (2000) Reliability of substance use disorder diagnoses among African-Americans and Caucasians. Drug Alcohol Depend 57(3):203-209

59. Demakis JG (2002) Preface to variation in health care (editorial). Med Care 40(Suppl 1):I1-I2

60. US Surgeon General (2001) Mental Health: Culture, Race, and Ethnicity. Substance Abuse and Mental Health Services Administration, Rockville, MD

61. National Quality Research Center (2003) American Consumer Satisfaction Index. University of Michigan, Ann Arbor, MI

62. Blow FC, McCarthy JF, Valenstein M, Zeber JE, Gillon L (2000) Care in the VHA for Veterans with Psychosis 1999. VHA Health Services Research \& Development, Ann Arbor

63. Gureje O (2002) Psychological disorders and symptoms in primary care. Association with disability and service use after 12 months. Soc Psychiatry Psychiatr Epidemiol 37(5):220-224

64. Ries RK, Comtois KA (1997) Illness severity and treatment services for dually diagnosed severely mentally ill outpatients. Schizophr Bull 23(2):239-246

65. Nease DE, Volk RJ, Cass AR (1999) Does the severity of mood and anxiety symptoms predict health care utilization? J Fam Pract 48(10):769-777

66. Rosenheck R, Massari L (1993) Wartime military service and utilization of VA health care services. Mil Med 158 (4):223-228

67. Munley PH, Vacha-Haase T, Busby RM, Paul BD (1998) The MCMI-II and race. J Pers Assess 70(1):183-189

68. Brown ES, Nejtek VA, Perantie DC, Bobadilla L (2002) Quetiapine in bipolar disorder and cocaine dependence. Bipolar Disord 4(6):406-411

69. Chengappa KN, Levine J, Gershon S, Kupfer DJ (2000) Lifetime prevalence of substance or alcohol abuse and dependence among subjects with bipolar I and II disorders in a voluntary registry. Bipolar Disord 2(3 Pt 1):191-195 
70. Strakowski SM, DelBello MP (2000) The co-occurrence of bipolar and substance use disorders. Clin Psychol Rev 20(2):191-206

71. Frueh BC, Brady KL, de Arellano MA (1998) Racial differences in combat-related PTSD: empirical findings and conceptual issues. Clin Psychol Rev 18(3):287-305

72. Rosenheck RA, Fontana A (1996) Race and outcome of treatment for veterans suffering from PTSD. J Trauma Stress 9(2):343-351

73. Ruef AM, Litz BT, Schlenger WE (2000) Hispanic ethnicity and risk for combat-related posttraumatic stress disorder. Cultur Divers Ethnic Minor Psychol 6(3):235-251

74. Frueh BC, Hamner MB, Bernat JA, Turner SM, Keane TM, Arana GW (2002) Racial differences in psychotic symptoms among combat veterans with PTSD. Depress Anxiety 16(4):157-161

75. Magruder KM, Frueh BC, Knapp RG, Johnson MR, Vaughan JA, Carson TC, Powell DA, Hebert R (in press) PTSD symptoms, demographic characteristics, and functional status among veterans treated in VA primary care clinics. J Trauma Stress

76. Magruder KM, Frueh BC, Knapp RG, Davis L, Hamner MB, Hebert R, Gold PB, Arana GW (under review) Prevalence of posttraumatic stress disorder in primary care clinics

77. Cooper-Patrick L, Gallo JJ, Gonzales JJ, Vu HT, Powe NR, Nelson C, Ford DE (1999) Race, gender, and partnership in the patientphysician relationship. JAMA 282(6):583-589

78. Copeland LA, Zeber JE, Valenstein M, Blow FC (2003) Racial disparity in the use of atypical antipsychotic medications among veterans. Am J Psychiatry 160(10):1817-1822

79. Harada ND, Damron-Rodriguez J, Villa VM, Washington DL, Dhanani S, Shon H, Chattapadhyay M, Fishbein H, Lee M, Makinodan T, et al. (2002) Veteran identity and race/ethnicity: influences on VA outpatient care utilization. Med Care 40(Suppl 1): I117-I128

80. Harada ND, Villa VM, Andersen R (2002) Satisfaction with VA and non-VA outpatient care among veterans. Am J Med Qual 17(4):155-164

81. Kazis LE, Ren XS, Lee A, Skinner K, Rogers W, Clark J, Miller DR (1999) Health status in VA patients: results from the Veterans Health Study. Am J Med Qual 14(1):28-38

82. Broman CL (1987) Race differences in professional help seeking. Am J Community Psychol 15(4):473-489

83. Diala CC, Muntaner C, Walrath C, Nickerson $\mathrm{K}$, LaVeist T, Leaf $\mathrm{P}$ (2001) Racial/ethnic differences in attitudes toward seeking professional mental health services. Am J Public Health 91(5): 805-807

84. Rosenheck R, Fontana A (1994) Utilization of mental health services by minority veterans of the Vietnam era. J Nerv Ment Dis 182(12):685-69

85. Washington DL, Harada ND, Villa VM, Damron-Rodriguez J, Dhanani S, Shon H, Makinodan T (2002) Racial variations in Department of Veterans Affairs ambulatory care use and unmet health care needs. Mil Med 167(3):235-241

86. Hoff RA, Rosenheck RA (2000) Cross-system service use among psychiatric patients: data from the Department of Veterans Affairs. J Behav Health Serv Res 27(1):98-106
87. Anand SS (1999) Using ethnicity as a classification variable in health research: perpetuating the myth of biological determinism, serving socio-political agendas, or making valuable contributions to medical sciences? Ethn Health 4(4):241-244

88. Krieger N (2000) Refiguring "race": epidemiology, racialized biology, and biological expressions of race relations. Int J Health Serv 30(1):211-216

89. Oppenheimer GM (2001) Paradigm lost: race, ethnicity, and the search for a new population taxonomy. Am J Public Health 91(7):1049-1055

90. Kressin NR, Chang B-H, Hendricks A, Kazis LE (2003) Agreement between administrative data and patients' self-reports of race/ethnicity. Am J Public Health 93(10):1734-1739

91. Hutchinson G, Takei N, Sham P, Harvey I, Murray RM (1999) Factor analysis of symptoms in schizophrenia: differences between White and Caribbean patients in Camberwell. Psychol Med 29(3):607-612

92. Weisman AG, Lopez SR, Ventura J, Nuechterlein KH, Goldstein MJ, Hwang S (2000) A comparison of psychiatric symptoms between Anglo-Americans and Mexican-Americans with schizophrenia. Schizophr Bull 26(4):817-824

93. Brekke JS, Barrio C (1997) Cross-ethnic symptom differences in schizophrenia: the influence of culture and minority status. Schizophr Bull 23(2):305-316

94. Kutchins H, Kirk SA (1989) DSM-III-R: the conflict over new psychiatric diagnoses. Health Soc Work 14(2):91-101

95. Cooksey EC, Brown P (1998) Spinning on its axes: DSM and the social construction of psychiatric diagnosis. Int J Health Serv 28(3):525-554

96. Sarbin TR (1990) Toward the obsolescence of the schizophrenia hypothesis. J Mind Behav 11(3-4):259-284

97. Westermeyer J (1985) Psychiatric diagnosis across cultural boundaries. Am J Psychiatry 142(7):798-805

98. Jampala V, Chowdry S, Taylor MA (1988) The use of DSM-III in the United States: a case of not going by the book. Compr Psychiatry 130:39-47

99. Manson SM, Kleinman A (1998) DSM-IV, culture and mood disorders: a clinical reflection on recent progress. Transcultural Psychiatry 35(3):377-386

100. Neighbors HW, Jackson JS, Campbell L, Williams D (1989) The influence of racial factors on psychiatric diagnosis: A review and suggestions for research. Community Ment Health J 25(4):301-311

101. Rosenheck R, Fontana A, Cottrol C (1995) Effect of clinicianveteran racial pairing in the treatment of posttraumatic stress disorder. Am J Psychiatry 152(4):555-563

102. Sterling RC, Gottheil E, Weinstein SP, Serota R (2001) The effect of therapist/patient race- and sex-matching in individual treatment. Addiction 96(7):1015-1022

103. Mathews CA, Glidden D, Hargreaves WA (2002) The effect on diagnostic rates of assigning patients to ethnically focused inpatient psychiatric units. Psychiatr Serv 53(7):823-829

104. Zuvekas SH, Taliaferro GS (2003) Pathways to access: health insurance, the health care delivery system, and racial/ethnic disparities, 1996-1999. Med Care 22(2):139-153 